\title{
INFLUENCE OF DISTILLATION APPARATUS AND DISTILLATION TIME ON THE YIELD AND CHEMICAL COMPOSITION OF WINTER SAVORY ESSENTIAL OIL
}

\section{WPŁYW APARATU DO DESTYLACJI I CZASU DESTYLACJI NA ILOŚĆ I SKŁAD CHEMICZNY OLEJKU ETERYCZNEGO Z CZĄBRU GÓRSKIEGO}

\author{
Department of Organic and Physical Chemistry, West Pomeranian University of Technology, \\ Szczecin, Poland \\ ${ }^{1}$ Department of Horticulture, West Pomeranian University of Technology, Szczecin, Poland
}

\begin{abstract}
Streszczenie. Celem badań prowadzonych w latach 2014-2015 było określenie wpływu aparatu do destylacji, jak również czasu destylacji na ilość i skład chemiczny olejku eterycznego z cząbru górskiego (Satureja montana L.). Olejek eteryczny wyizolowano z ziela S. montana, zebranego w okresie kwitnienia, wykorzystując dwa różne aparaty do destylacji: Derynga i Clevengera. Ponadto zastosowano różny czas destylacji (2, 3 i 4 godziny). Otrzymane olejki zanalizowano, wykorzystując chromatograf gazowy sprzężony ze spektrometrem masowym (GC-MS). Analiza GC-MS wykazała obecność 47 związków w olejkach otrzymanych zarówno w aparacie Derynga, jak i w aparacie Clevengera. Głównym składnikiem wszystkich analizowanych próbek olejku S. montana był karwakrol $(54,44-68,53 \%)$. Innymi ważnymi składnikami były: $\gamma$-terpinen $(5,21-8,67 \%)$, p-cymen $(4,40-9,53 \%)$, a-terpinen $(1,20-2,04 \%)$ oraz $\beta$-kariofilen $(4,58-5,77 \%)$. Na podstawie uzyskanych wyników badań stwierdzono, że czas destylacji w aparacie Derynga nie miał istotnego wpływu na zawartość olejku. Natomiast zawartość olejku uzyskanego w aparacie Clevengera była zależna od czasu destylacji. Ponadto nie wykazano wpływu czasu destylacji na ilość głównych składników zidentyfikowanych w olejkach wydzielonych w obu aparatach do destylacji, chociaż odnotowano znaczące interakcje pomiędzy składnikiem olejku a czasem destylacji.
\end{abstract}

Key words: carvacrol, distillation apparatus, essential oil composition, GC-MS, hydrodistillation, Satureja montana L.

Słowa kluczowe: aparat do destylacji, GC-MS, hydrodestylacja, karwakrol, Satureja montana L., skład olejku eterycznego.

\section{INTRODUCTION}

Satureja montana L. (winter savory or mountain savory), belongs to the Lamiaceae family, native to warm temperate regions of southern Europe (Damjanovic-Vratnica et al. 2011). This well-known bushy perennial subshrub with woody stems at the base, linear leaves and pale pink flowers (Lawless 2002) is frequently used as traditional medicinal herb and spice for food and teas (Skocibusic and Bezic 2004). The leaves and flowering tops of this strong aromatic plant are used as a seasoning for meats and fish as well as flavoring agent for

Corresponding author - Adres do korespondencji: Aneta Wesołowska Department of Organic and Physical Chemistry, West Pomeranian University of Technology, Szczecin, Piastów 42, 71-065 Szczecin, Poland, e-mail: Aneta.Wesolowska@zut.edu.pl 
soups, sausages, canned meats, and spicy sauces (Miladi et al. 2013). Savory honey is a popular ingredient in folk remedies for the treatment of bronchitis and premature ejaculation (Zavatti et al. 2011). The whole herb, and especially the flowering shoots, is mildly antiseptic, carminative, digestive, mildly expectorant and stomachic (Hassanein et al. 2014).

The biological properties of winter savory are related to the presence of thymol, carvacrol p-cymene, $\gamma$-terpinene and $\beta$-caryophyllene (Ruberto and Baratta 2000; Radonic and Milos 2003; Skocibusic and Bezic 2004) in its essential oil. High antioxidant, antibacterial and antimicrobial activity of ground material, volatile oil and extracts of winter savory has been well documented (Valnet 1990; Madsen et al. 1996; Pepeljak et al. 1999; Ciani et al. 2000; Cavar et al. 2008; Serrano et al. 2011).

Commercially, winter savory oil is used to flavor condiments, relishes, soups, sauces, sausages, and prepared meats (Small and Deutsch 2001). Occasionally it is used in perfumery (Lawless 2002). The oil and savory extracts may be also used as natural antioxidants and antimicrobials in the preservation of processed food (Serrano et al. 2011) and cosmetics (Chorianopoulos et al. 2004).

The chemical composition of $S$. montana essential oil as well as concentration of biologically active compounds depend on many factors, such as environmental conditions, harvest term, geographic origin, and storage conditions (Milos et al. 2001).

Our previous research indicated that the optimal time for collecting the savory plants with respect to high carvacrol, $y$-terpinene and $\beta$-caryophyllene content is before flowering, while plants collected after the flowering gave oil rich in p-cymene (Wesołowska et al. 2014).

According to literature data the high carvacrol content determines the value of savory oil (Lawrence 1979), while its biological properties are attributed to the presence of carvacrol and p-cymene (Skocibusic and Bezic 2004).

Therefore, we decided to investigate the influence of distillation apparatus and distillation time on the yield and chemical composition of winter savory (Satureja montana L.) essential oil, especially on the relative percentage of carvacrol. To the best of our knowledge, there are no scientific data concerned with this topic.

\section{MATERIAL AND METHODS}

Plant material. The field experiment was carried out at the Horticultural Experimental Station in Dołuje (near Szczecin, north-western Poland), which belongs to the Department of Horticulture of the West Pomeranian University of Technology in Szczecin.

The experiment was performed on sandy clay soil which is characterized by low water-holding capacity. The field was prepared according to agrotechnique proper for perennial plants from Lamiaceae family. Mineral fertilization was quantified according to the results of the chemical analysis of the soil samples. In the first year nitrogen fertilizer $\left(80 \mathrm{~kg} \mathrm{~N} \mathrm{ha}^{-1}\right)$ was applied in three equal doses: $1 / 3$ before sowing, $1 / 3$ three weeks after sowing and $1 / 3$ after harvest. Phosphorous fertilizer $\left(60 \mathrm{~kg} \mathrm{P}_{2} \mathrm{O}_{5} \cdot \mathrm{ha}^{-1}\right)$ and potassium fertilizer $\left(100 \mathrm{~kg} \mathrm{~K}_{2} \mathrm{O} \cdot \mathrm{ha}^{-1}\right)$ were applied once, during spring cultivation treatment. In further years of cultivation the whole phosphorous fertilizer and potassium fertilizer were applied once (on early spring) before plants vegetation. Nitrogen fertilizer was applied in two doses: $1 / 2$ before plants vegetation and $1 / 2$ after harvest. During the growing season manual weeding and irrigation were performed. 
The savory seeds (purchased from Herb Factory 'Kawon-Hurt', Gostyń, Poland) were sown on seedbed at the first decade of April 2013. In the same year, the obtained seedlings were planted into the open field in the first decade of June, with spacing of $40 \times 30 \mathrm{~cm}$. The plants were grown in experimental plots with an area of $1.44 \mathrm{~m}^{2}$, in four replications. For laboratory analyses a herb from 2- and 3-year-old plants was collected during flowering (harvest dates: 7 August 2014 and 18 August 2015). A $100 \mathrm{~g}$ samples, from each experimental plot $(4 \times 100 \mathrm{~g})$, were taken.

Subsequently, the samples of plant material were combined, mixed and dried in a shady and well ventilated place at room temperature (drying room). Dry herb was rubbed through the sieve of mesh diameter 4-5 mm, placed in paper bags and stored in a dry and cool place until analysis (within two months the chemical analysis of dried plant material was performed). Representative samples (200 g, each year) were used for essential oil isolation.

Essential oil isolation. Ten grams of dried aerial parts of winter savory in a $1000 \mathrm{ml}$ round-bottomed flask along with $500 \mathrm{ml}$ of distilled water was subjected to hydrodistillation using Deryng apparatus recommended by Polish Pharmacopoeia VI (2002). The hydrodistillation was also performed with use of Clevenger apparatus recommended by Polish Pharmacopeia VIII (2008) as well as European Pharmacopoeia 5.0 (2004). Moreover, three different distillation times: 2, 3 and 4 hours were applied. Isolated essential oils were dried over anhydrous sodium sulphate and, after filtration, stored in dark sealed vials at $4^{\circ} \mathrm{C}$ until GC-MS analysis. Tree replicates were carried out. Essential oil percentage was calculated based on dry weight of plant material and expressed as (\% v/w) in Table 1.

Analysis of essential oils composition. The qualitative GC-MS analysis of the obtained volatile oils was carried out using an HP 6890 gas chromatograph equipped with a HP-5MS capillary column ( $30 \mathrm{~m} \times 0.25 \mathrm{~mm}$, film thickness $0.25 \mu \mathrm{m})$ and coupled with HP 5973 Mass Selective Detector.

The flow rate of helium (carrier gas) through the column was kept at $1.0 \mathrm{ml} \cdot \mathrm{min}^{-1}$. Samples of $2 \mu \mathrm{l}$ (30 $\mathrm{mg}$ of oil dissolved in $1.5 \mathrm{ml}$ of dichloromethane) were injected with a split ratio of $5: 1$. The injector and the transfer line were kept at $280^{\circ} \mathrm{C}$. The ion source temperature was $230^{\circ} \mathrm{C}$.

The initial temperature of the column was $40^{\circ} \mathrm{C}$ for 5 minutes, then increased to $60^{\circ} \mathrm{C}$ at a rate of $30^{\circ} \mathrm{C}$ per minute, next to $230^{\circ} \mathrm{C}$ at a rate of $6^{\circ} \mathrm{C}$ per minute (kept constant for $10 \mathrm{~min}$ ), and then increased to a final temperature of $280^{\circ} \mathrm{C}$ at a rate of $30^{\circ} \mathrm{C}$ per minute. The oven was held at this temperature for 5 minutes. Mass spectra were taken at $70 \mathrm{eV}$. Mass range was from 40 to $550 \mathrm{~m} / \mathrm{z}$.

Solvent delay was 4 minutes. The total running time for a sample was about 51 minutes.

The relative percentage amounts of the essential oil constituents were evaluated from the total peak area (TIC) using apparatus software.

Essential oil constituents were identified by comparison of their retention indices (relative to n-alkanes $\mathrm{C}_{7}-\mathrm{C}_{40}$ (Supelco, Bellefonte, PA, USA) on HP-5MS column) with those reported in NIST Chemistry WebBook (http://webbook.nist.gov/chemistry/) and literature (Miladi et al. 2013). Further identification was made by comparison of their mass spectra with those stored in the Wiley NBS75K.L and NIST/EPA/NIH (2002 and 2014 version) mass spectral libraries, as well as by comparison of their mass spectra with authentic compounds available in our laboratory (thymol, carvacrol, $p$-cymene and $\beta$-pinene), purchased from Fluka and Sigma-Aldrich. 
Statistical analysis. The results of the study were subjected to an analysis of variance which was performed with AWAR software, made by the Department of Agrometeorology and Applied Informatics, Institute of Soil Science and Plant Cultivation in Puławy, Poland (Filipiak and Wilkos 1995). The means of two years were separated using the Tukey's Studentized Range Test at $p=0.05$. The statistical analysis of the results given in Table 4 and 5 was conducted for constituents of content greater than $1 \%$ of the essential oil.

\section{RESULTS}

The essential oils obtained from the aerial parts of Satureja montana L. using two types of distillation apparatuses at different distillation times, were found to be yellow-orange liquids with percentage content shown in Table 1.

Table 1. Essential oil content in winter savory herb in dependence on distillation time and distillation apparatus

Tabela 1. Zawartość olejku eterycznego w zielu cząbru górskiego w zależności od czasu destylacji i aparatu do destylacji

\begin{tabular}{lcccccc}
\hline & \multicolumn{4}{c}{ Essential oil content - Zawartość olejku eterycznego [\% v/w] } \\
\cline { 2 - 7 } $\begin{array}{l}\text { Distillation time } \\
\text { Czas destylacji }\end{array}$ & \multicolumn{3}{c}{$\begin{array}{c}\text { Deryng apparatus } \\
\text { aparat Derynga }\end{array}$} & \multicolumn{3}{c}{$\begin{array}{c}\text { Clevenger apparatus } \\
\text { aparat Clevengera }\end{array}$} \\
\cline { 2 - 7 } & 2014 & 2015 & $2014-2015$ & 2014 & 2015 & $2014-2015$ \\
\hline $2 \mathrm{~h}$ & $1.80 \pm 0.08$ & $2.93 \pm 0.05$ & 2.37 & $1.37 \pm 0.09$ & $2.20 \pm 0.14$ & 1.79 \\
$3 \mathrm{~h}$ & $1.76 \pm 0.05$ & $2.70 \pm 0.25$ & 2.34 & $1.50 \pm 0.00$ & $2.47 \pm 0.13$ & 1.99 \\
$4 \mathrm{~h}$ & $1.83 \pm 0.25$ & $3.00 \pm 0.00$ & 2.42 & $1.70 \pm 0.08$ & $2.30 \pm 0.08$ & 2.00 \\
Mean - Średnia & 1.80 & 2.88 & 2.34 & 1.52 & 2.32 & 1.92 \\
LSD $_{\alpha=0,05}$ & n.s. & n.s. & n.s. & 0.307 & n.s. & 0.215 \\
NIR $=0,05$ & & & & & \\
\hline
\end{tabular}

n.s. - not significant - różnice nieistotne.

\pm - standard deviation - średnia \pm odchylenie standardowe $(n=3)$.

The presented data indicate that the time of essential oil distillation of winter savory herb in Deryng apparatus had no significant effect on its content. The lack of the significant differences was noted for 2-year-old and 3-year old material. The amounts of essential oil was $1.80 \%$ in 2014 and $2.88 \%$ in 2015 . The amounts obtained from the Clevenger apparatus were slightly lower (respectively: $1.52 \%$ and $2.32 \%$ ). Moreover, it was shown that the amount of essential oil distilled in the Clevenger apparatus was significantly influenced by the distillation time. The significant differences were noted for the first year of study and for the synthesis of both years of the study. The more effective distillation time was 3 and 4 hours, in comparison with the 2 hour distillation.

The identified components of the S. montana oils are listed in Table 2 and 3, in which the relative percentage and retention indices of the constituents are given.

A total of 47 different constituents were identified in the essential oils obtained by distillation in Deryng apparatus (Table 2). 
Table 2. Percentage composition of winter savory essential oils in dependence on distillation time in Deryng apparatus

Tabela 2. Skład procentowy olejków eterycznych z cząbru górskiego w zależności od czasu destylacji w aparacie Derynga

\begin{tabular}{|c|c|c|c|c|c|c|c|}
\hline \multirow{2}{*}{$\begin{array}{l}\text { Components } \\
\text { Składniki }\end{array}$} & \multirow{2}{*}{$\mathrm{RI}$} & \multicolumn{2}{|c|}{$2 \mathrm{~h}$} & \multicolumn{2}{|c|}{$3 \mathrm{~h}$} & \multicolumn{2}{|c|}{$4 \mathrm{~h}$} \\
\hline & & 2014 & 2015 & 2014 & 2015 & 2014 & 2015 \\
\hline$\alpha$-Thujene $-\alpha$-Tujen & 928 & 0.99 & 0.44 & 0.86 & 0.52 & 0.85 & 0.45 \\
\hline$\alpha$-Pinene - $\alpha$-Pinen & 934 & 0.77 & 0.55 & 0.67 & 0.64 & 0.63 & 0.57 \\
\hline Camphene - Kamfen & 949 & 0.19 & 0.08 & 0.17 & 0.10 & 0.15 & 0.09 \\
\hline$\beta$-Pinene - $\beta$-Pinen & 977 & 0.22 & 0.15 & 0.19 & 0.17 & 0.20 & 0.16 \\
\hline 1-Octen-3-ol - 1-Okten-3-ol & 981 & 0.15 & - & 0.14 & - & 0.15 & - \\
\hline Myrcene - Mircen & 992 & 1.42 & 0.62 & 1.37 & 0.82 & 1.39 & 0.78 \\
\hline Decane - Dekan & 999 & - & 0.12 & - & 0.11 & - & 0.09 \\
\hline$\alpha$-Phellandrene - $\alpha$-Felandren & 1005 & 0.32 & 0.30 & 0.30 & 0.32 & 0.29 & 0.30 \\
\hline 3-Carene-3-Karen & 1011 & 0.11 & 0.14 & 0.11 & 0.14 & 0.09 & 0.12 \\
\hline$\alpha$-Terpinene - $\alpha$-Terpinen & 1017 & 2.04 & 1.50 & 1.96 & 1.69 & 1.93 & 1.56 \\
\hline p-Cymene - p-Cymen & 1025 & 9.53 & 5.06 & 8.76 & 5.68 & 8.99 & 5.41 \\
\hline$\beta$-Phellandrene - $\beta$-Felandren & 1029 & 0.71 & - & 0.68 & 0.19 & 0.66 & 0.17 \\
\hline Eucalyptol - Eukaliptol & 1032 & 1.65 & 0.85 & 1.51 & 0.83 & 1.32 & 0.72 \\
\hline (Z)- $\beta$-Ocimene - (Z)- $\beta$-Ocymen & 1037 & - & 0.81 & - & 0.76 & - & 0.48 \\
\hline$y$-Terpinene $-y$-Terpinen & 1060 & 8.67 & 6.30 & 8.46 & 6.84 & 8.42 & 6.62 \\
\hline cis-Sabinene hydrate - cis-Hydrat Sabinenu & 1069 & 1.17 & 1.48 & 1.17 & 1.38 & 1.03 & 1.39 \\
\hline$\alpha$-Terpinolene $-\alpha$-Terpinolen & 1090 & 0.12 & 0.03 & 0.12 & 0.04 & - & 0.03 \\
\hline Undecane - Undekan & 1101 & 0.18 & 0.04 & 0.19 & 0.02 & 0.19 & 0.04 \\
\hline Linalool - Linalol & 1107 & 0.32 & 0.18 & 0.32 & 0.19 & 0.30 & 0.22 \\
\hline Borneol - Borneol & 1169 & 0.53 & 0.11 & 0.53 & 0.20 & 0.54 & 0.12 \\
\hline$\alpha$-Phellandren-8-ol - $\alpha$-Felandren-8-ol & 1172 & - & 0.10 & - & 0.03 & - & 0.11 \\
\hline Terpinen-4-ol - Terpinen-4-ol & 1183 & 0.75 & 0.63 & 0.76 & 0.66 & 0.77 & 0.62 \\
\hline$\alpha$-Terpineol - $\alpha$-Terpineol & 1196 & 0.21 & 0.12 & 0.21 & 0.15 & 0.19 & 0.13 \\
\hline Thymoquinone - Tymochinon & 1254 & 2.01 & 0.44 & 1.95 & 0.47 & 2.25 & 0.65 \\
\hline Thymol - Tymol & 1294 & 0.35 & 0.40 & 0.36 & 0.40 & 0.37 & 0.41 \\
\hline Carvacrol - Karwakrol & 1310 & 57.10 & 68.53 & 56.55 & 64.28 & 55.20 & 63.39 \\
\hline 3-Methyltridecane - 3-Metylotridekan & 1377 & 0.04 & 0.23 & 0.05 & 0.21 & - & 0.18 \\
\hline a-Copaene - a-Kopaen & 1381 & 0.15 & 0.39 & 0.18 & 0.34 & 0.18 & 0.35 \\
\hline$\beta$-Bourbonene $-\beta$-Burbonen & 1390 & 0.07 & 0.16 & 0.07 & 0.19 & 0.09 & 0.12 \\
\hline a-Gurjunene - a-Gurjunen & 1415 & 0.08 & 0.18 & 0.08 & 0.19 & 0.10 & 0.21 \\
\hline$\beta$-Caryophyllene $-\beta$-Kariofilen & 1427 & 4.58 & 4.94 & 5.09 & 5.32 & 5.54 & 5.59 \\
\hline$\beta$-Cubebene - $\beta$-Kubeben & 1436 & 0.14 & 0.18 & 0.16 & 0.21 & 0.18 & 0.21 \\
\hline Aromadendrene - Aromadendren & 1446 & 0.27 & 0.37 & 0.32 & 0.42 & 0.36 & 0.47 \\
\hline$\alpha$-Humulene - $\alpha$-Humulen & 1461 & 0.20 & 0.22 & 0.21 & 0.24 & 0.23 & 0.26 \\
\hline y-Muurolene - $\mathrm{y}$-Muurolen & 1482 & 0.38 & 0.47 & 0.44 & 0.56 & 0.50 & 0.64 \\
\hline Germacrene D - Germakren D & 1488 & 0.26 & 0.18 & 0.34 & 0.19 & 0.37 & 0.24 \\
\hline Viridiflorene - Wiridifloren & 1494 & 0.10 & 0.05 & 0.10 & 0.11 & 0.13 & 0.11 \\
\hline Bicyclogermacrene - Bicyklogermakren & 1503 & 0.56 & 0.62 & 0.67 & 0.67 & 0.75 & 0.71 \\
\hline a-Muurolene - a-Muurolen & 1505 & - & - & - & 0.08 & - & 0.13 \\
\hline$\beta$-Bisabolene $-\beta$-Bisabolen & 1513 & 0.75 & 0.43 & 1.03 & 0.72 & 1.29 & 0.88 \\
\hline$y$-Cadinene - y-Kadinen & 1521 & 0.41 & 0.45 & 0.50 & 0.59 & 0.53 & 0.68 \\
\hline$\delta$-Cadinene $-\delta$-Kadinen & 1529 & 0.58 & 0.76 & 0.71 & 0.96 & 0.78 & 1.10 \\
\hline Spathulenol - Spatulenol & 1588 & 0.15 & 0.03 & 0.18 & 0.09 & 0.22 & 0.08 \\
\hline Caryophyllene oxide - Tlenek kariofilenu & 1593 & 1.20 & 0.54 & 1.25 & 0.56 & 1.32 & 0.56 \\
\hline Isophytol - Izofitol & 1941 & - & 0.09 & - & 0.35 & - & 0.48 \\
\hline Linolenic acid - Kwas linolenowy & 2148 & 0.30 & - & 0.71 & 0.74 & 0.84 & 1.31 \\
\hline 1-Nonadecanol - 1-Nonadekanol & 2165 & 0.12 & 0.25 & 0.38 & 0.51 & 0.41 & 0.69 \\
\hline Identified - Zidentyfikowano & & 99.85 & 99.52 & 99.81 & 99.88 & 99.73 & 99.63 \\
\hline $\begin{array}{l}\text { Monoterpene hydrocarbons } \\
\text { Węglowodory monoterpenowe }\end{array}$ & & 25.09 & 15.98 & 23.65 & 17.91 & 23.60 & 16.74 \\
\hline $\begin{array}{l}\text { Oxygenated monoterpenes } \\
\text { Utlenione monoterpeny }\end{array}$ & & 64.09 & 72.84 & 63.36 & 68.59 & 61.97 & 67.76 \\
\hline $\begin{array}{l}\text { Sesquiterpene hydrocarbons } \\
\text { Węglowodory seskwiterpenowe }\end{array}$ & & 8.53 & 9.40 & 9.90 & 10.79 & 11.03 & 11.70 \\
\hline $\begin{array}{l}\text { Oxygenated sesquiterpenes } \\
\text { Utlenione seskwiterpeny }\end{array}$ & & 1.35 & 0.57 & 1.43 & 0.65 & 1.54 & 0.64 \\
\hline $\begin{array}{l}\text { Oxygenated diterpenes } \\
\text { Utlenione diterpeny }\end{array}$ & & - & 0.09 & - & 0.35 & - & 0.48 \\
\hline Others - Inne & & 0.79 & 0.64 & 1.47 & 1.59 & 1.59 & 2.31 \\
\hline
\end{tabular}

$\mathrm{RI}$ - retention index - indeks retencji.

"-" - not found - nie wykryto. 
Table 3. Percentage composition of winter savory essential oils in dependence on distillation time in Clevenger apparatus

Tabela 3. Skład procentowy olejków eterycznych z cząbru górskiego w zależności od czasu destylacji w aparacie Clevengera

\begin{tabular}{|c|c|c|c|c|c|c|c|}
\hline \multirow{2}{*}{$\begin{array}{l}\text { Components } \\
\text { Składniki }\end{array}$} & \multirow{2}{*}{ RI } & \multicolumn{2}{|c|}{$2 \mathrm{~h}$} & \multicolumn{2}{|c|}{$3 \mathrm{~h}$} & \multicolumn{2}{|c|}{$4 \mathrm{~h}$} \\
\hline & & 2014 & 2015 & 2014 & 2015 & 2014 & 2015 \\
\hline$\alpha$-Thujene - $\alpha$-Tujen & 928 & 0.77 & 0.39 & 0.77 & 0.30 & 0.72 & 0.26 \\
\hline$\alpha$-Pinene - $\alpha$-Pinen & 934 & 0.62 & 0.48 & 0.60 & 0.38 & 0.55 & 0.34 \\
\hline Camphene - Kamfen & 949 & 0.16 & 0.08 & 0.15 & 0.04 & 0.14 & 0.04 \\
\hline$\beta$-Pinene $-\beta$-Pinen & 977 & 0.20 & 0.14 & 0.19 & 0.09 & 0.19 & 0.12 \\
\hline 1-Octen-3-ol - 1-Okten-3-ol & 981 & 0.15 & - & 0.14 & - & 0.18 & - \\
\hline Myrcene - Mircen & 992 & 1.29 & 0.59 & 1.30 & 0.49 & 1.34 & 0.47 \\
\hline Decane - Dekan & 999 & - & 0.13 & - & 0.05 & - & 0.11 \\
\hline a-Phellandrene - $\alpha$-Felandren & 1005 & 0.29 & 0.29 & 0.28 & 0.27 & 0.28 & 0.25 \\
\hline 3-Carene - 3-Karen & 1011 & 0.11 & 0.14 & 0.09 & 0.13 & 0.10 & 0.11 \\
\hline$\alpha$-Terpinene $-\mathrm{y}$-Terpinen & 1017 & 1.88 & 1.47 & 1.96 & 1.27 & 1.90 & 1.20 \\
\hline p-Cymene - p-Cymen & 1025 & 9.38 & 5.05 & 9.23 & 4.40 & 9.13 & 4.79 \\
\hline$\beta$-Phellandrene - $\beta$-Felandren & 1029 & 0.43 & - & 0.66 & - & 0.67 & - \\
\hline Eucalyptol - Eukaliptol & 1032 & 1.43 & 0.81 & 1.28 & 0.80 & 1.53 & 0.81 \\
\hline (Z)- $\beta$-Ocimene - (Z)- $\beta$-Ocymen & 1037 & - & 0.78 & - & 0.81 & - & 0.70 \\
\hline $\mathrm{y}$-Terpinene $-\mathrm{y}$-Terpinen & 1060 & 8.22 & 6.23 & 8.75 & 5.49 & 8.30 & 5.21 \\
\hline cis-Sabinene hydrate - cis-Hydrat sabinenu & 1069 & 1.15 & 1.39 & 1.12 & 1.49 & 1.06 & 1.32 \\
\hline$\alpha$-Terpinolene - $\alpha$-Terpinolen & 1090 & 0.12 & - & 0.11 & - & 0.13 & - \\
\hline Undecane - Undekan & 1101 & 0.24 & 0.03 & 0.23 & 0.03 & 0.27 & 0.03 \\
\hline Linalool - Linalol & 1107 & 0.30 & 0.19 & 0.27 & 0.21 & 0.35 & 0.18 \\
\hline Borneol - Borneol & 1169 & 0.57 & 0.12 & 0.54 & 0.11 & 0.56 & 0.12 \\
\hline a-Phellandren-8-ol - $\alpha$-Felandren-8-ol & 1172 & - & 0.11 & - & 0.10 & - & 0.11 \\
\hline Terpinen-4-ol - Terpinen-4-ol & 1183 & 0.82 & 0.59 & 0.81 & 0.58 & 0.81 & 0.61 \\
\hline$\alpha$-Terpineol - $\alpha$-Terpineol & 1196 & 0.28 & 0.13 & 0.27 & 0.12 & 0.22 & 0.14 \\
\hline $\begin{array}{l}\text { trans-Dihydrocarvone - trans } \\
\text { Dihydrokarwon }\end{array}$ & 1202 & 0.11 & - & 0.10 & - & 0.10 & - \\
\hline Thymoquinone - Tymochinon & 1254 & 2.55 & 0.74 & 2.06 & 0.65 & 2.42 & 0.80 \\
\hline Thymol - Tymol & 1294 & 0.37 & 0.38 & 0.36 & 0.36 & 0.37 & 0.37 \\
\hline Carvacrol - Karwakrol & 1310 & 56.65 & 66.95 & 56.54 & 66.06 & 54.44 & 65.33 \\
\hline 3-Methyltridecane - 3-Metylotridekan & 1377 & 0.06 & 0.16 & 0.05 & 0.22 & 0.03 & 0.16 \\
\hline a-Copaene - a-Kopaen & 1381 & 0.16 & 0.31 & 0.17 & 0.41 & 0.19 & 0.31 \\
\hline$\beta$-Bourbonene - $\beta$-Burbonen & 1390 & 0.07 & 0.11 & 0.08 & 0.18 & 0.09 & 0.13 \\
\hline$\alpha$-Gurjunene - $\alpha$-Gurjunen & 1415 & 0.09 & 0.16 & 0.09 & 0.22 & 0.11 & 0.18 \\
\hline$\beta$-Caryophyllene - $\beta$-Kariofilen & 1427 & 4.88 & 5.26 & 5.09 & 5.74 & 5.52 & 5.77 \\
\hline$\beta$-Cubebene $-\beta$-Kubeben & 1436 & 0.14 & 0.19 & 0.16 & 0.23 & 0.18 & 0.20 \\
\hline Aromadendrene - Aromadendren & 1446 & 0.29 & 0.40 & 0.32 & 0.46 & 0.36 & 0.46 \\
\hline a-Humulene - $\alpha$-Humulen & 1461 & 0.21 & 0.25 & 0.21 & 0.28 & 0.25 & 0.27 \\
\hline Y-Muurolene - y-Muurolen & 1482 & 0.41 & 0.55 & 0.45 & 0.60 & 0.52 & 0.60 \\
\hline Germacrene D - Germakren D & 1488 & 0.28 & 0.24 & 0.31 & 0.25 & 0.36 & 0.22 \\
\hline Viridiflorene - Wiridifloren & 1494 & 0.10 & 0.10 & 0.11 & 0.10 & 0.13 & 0.13 \\
\hline Bicyclogermacrene - Bicyklogermakren & 1503 & 0.62 & 0.66 & 0.66 & 0.80 & 0.77 & 0.84 \\
\hline$\beta$-Bisabolene $-\beta$-Bisabolen & 1513 & 0.93 & 0.60 & 1.00 & 0.80 & 1.33 & 0.98 \\
\hline y-Cadinene - y-Kadinen & 1521 & 0.47 & 0.54 & 0.50 & 0.61 & 0.55 & 0.69 \\
\hline $\bar{\delta}$-Cadinene - $\delta$-Kadinen & 1529 & 0.65 & 0.90 & 0.69 & 1.01 & 0.81 & 1.10 \\
\hline Spathulenol - Spatulenol & 1588 & 0.24 & 0.08 & 0.20 & 0.11 & 0.26 & 0.12 \\
\hline Caryophyllene oxide - Tlenek kariofolenu & 1593 & 1.38 & 0.52 & 1.16 & 0.61 & 1.36 & 0.65 \\
\hline Isophytol - Izofitol & 1941 & - & 0.34 & - & 0.35 & - & 0.55 \\
\hline Linolenic acid - Kwas linolenowy & 2148 & 0.59 & 0.85 & 0.60 & 1.36 & 0.80 & 1.78 \\
\hline 1-Nonadecanol - 1-Nonadekanol & 2165 & 0.30 & 0.39 & 0.27 & 0.82 & 0.38 & 0.87 \\
\hline Identified - Zidentyfikowano & & 99.96 & 99.82 & 99.93 & 99.39 & 99.76 & 99.43 \\
\hline $\begin{array}{l}\text { Monoterpene hydrocarbons } \\
\text { Węglowodory monoterpenowe }\end{array}$ & & 23.47 & 15.64 & 24.09 & 13.67 & 23.45 & 13.49 \\
\hline $\begin{array}{l}\text { Oxygenated monoterpenes } \\
\text { Utlenione monoterpeny }\end{array}$ & & 64.23 & 71.41 & 63.35 & 70.48 & 61.86 & 69.79 \\
\hline $\begin{array}{l}\text { Sesquiterpene hydrocarbons } \\
\text { Węglowodory seskwiterpenowe }\end{array}$ & & 9.30 & 10.27 & 9.84 & 11.69 & 11.17 & 11.88 \\
\hline $\begin{array}{l}\text { Oxygenated sesquiterpenes } \\
\text { Utlenione seskwiterpeny }\end{array}$ & & 1.62 & 0.60 & 1.36 & 0.72 & 1.62 & 0.77 \\
\hline $\begin{array}{l}\text { Oxygenated diterpenes } \\
\text { Utlenione diterpeny }\end{array}$ & & - & 0.34 & - & 0.35 & - & 0.55 \\
\hline Others - Inne & & 1.34 & 1.56 & 1.29 & 2.48 & 1.66 & 2.95 \\
\hline
\end{tabular}


The main components were carvacrol (55.20-68.53\%), y-terpinene (6.30-8.67\%), p-cymene (5.06-9.53\%), $\beta$-caryophyllene (4.58-5.59\%), thymoquinone (0.44-2.25\%), $\alpha$-terpinene $(1.50-2.04 \%)$ and cis-sabinene hydrate (1.03-1.48\%). Interestingly, 1-octen-3-ol (0.14-0.15\%) was found only in the volatile oil obtained from plants harvested in 2014 , while decane (0.09-0.12\%), (Z)- $\beta$-ocimene (0.48-0.81\%), $\alpha$-phellandren-8-ol $(0.03-0.11 \%)$ and isophytol $(0.09-0.48 \%)$ were present only in the oil of plants collected in 2015 . Moreover, a-muurolene was detected only in the essential oils obtained by distillation (conducted by three and four hours) of plants collected in 2015.

The isolated oils were rich in oxygenated monoterpenes (61.97-72.84\%), monoterpene hydrocarbons (15.98-25.09\%) and sesquiterpene hydrocarbons (8.53-11.70\%) (Table 2).

The oils obtained by distillation in Clevenger apparatus consisted of 47 compounds in total (Table 3).

Similarly, carvacrol (54.44-66.95\%) was the main constituent of volatile oils, followed by $y$-terpinene (5.21-8.75\%), p-cymene (4.40-9.38\%), $\beta$-caryophyllene (4.88-5.77\%), thymoquinone (0.65-2.55\%), a-terpinene (1.20-1.96\%) and cis-sabinene hydrate (1.06-1.49\%). 1-Octen-3-ol $(0.14-0.18 \%)$ was also detected only in the oil isolated from savory herb collected in 2014. Likewise, decane (0.05-0.13\%), (Z)- $\beta$-ocimene $(0.70-0.81 \%)$, $\alpha$-phellandren-8-ol $(0.10-0.11 \%)$ and isophytol $(0.34-0.55 \%)$ were present only in the volatile oil of plants collected in 2015.

Interestingly, a-muurolene was not detected in the essential oils obtained by distillation in Clevenger apparatus, while trans-dihydrocarvone was not present in the oils obtained in Deryng apparatus.

The percentage of oxygenated monoterpenes (61.86-71.41\%) and monoterpene hydrocarbons (13.49-24.09\%) in savory oils obtained by distillation in Clevenger apparatus (Table 3) were found to be slightly lower as compared with the oils obtained in Deryng apparatus (Table 2). The amounts of sesquiterpene hydrocarbons were rather similar in both essential oils (8.53-11.79\% for Deryng and $9.30-11.88 \%$ for Clevenger, respectively).

The statistical analysis of the results given in Tables 4 and 5 showed that in each year of the study as well as the synthesis of both years of the study, there were significant differences between the content of the main winter savory herb essential oil constituents found.

The highest concentration was noted for carvacrol, then for $\mathrm{y}$-terpinene and p-cymene, and for $\beta$-caryophyllene. The content of the other constituents was significantly lower in comparison with the ones mentioned above. This pattern was noted for both distillation apparatuses. On average for both years of the study, the amount of carvacrol was $60.84 \%$ using the Deryng apparatus and $61.00 \%$ using the Clevenger apparatus, the amount of Y-terpinene was respectively: 7.55 and $7.03 \%$, p-cymene: 7.24 and $7.00 \%$, $\beta$-caryophyllene: 5.18 and $5.38 \%$. The amount of the other constituents of the essential oil distilled in the Deryng apparatus varied from 0.65 up to $1.78 \%$, while for distillation in the Clevenger apparatus - from 0.86 up to $1.61 \%$.

There was no significant effect of the distillation time on the amount of the main essential oil constituents obtained with both types of apparatuses. However, there was a significant interaction found between the constituent and the distillation time. 
Table 4. Content of main essential oil constituents in winter savory herb in dependence on distillation time in Deryng apparatus

Tabela 4. Zawartość głównych składników olejku eterycznego z cząbru górskiego w zależności od czasu destylacji w aparacie Derynga

\begin{tabular}{|c|c|c|c|c|c|c|c|c|c|c|c|c|}
\hline \multirow{3}{*}{$\begin{array}{c}\text { Essential oil constituent } \\
\text { (factor I) } \\
\text { Składnik olejku eterycznego } \\
\text { (czynnik I) }\end{array}$} & \multicolumn{12}{|c|}{ Distillation time (factor II) - Czas destylacji (czynnik II) } \\
\hline & \multicolumn{4}{|c|}{2014} & \multicolumn{4}{|c|}{2015} & \multicolumn{4}{|c|}{ 2014-2015 } \\
\hline & $2 \mathrm{~h}$ & $3 \mathrm{~h}$ & $4 \mathrm{~h}$ & $\begin{array}{c}\text { mean } \\
\text { śre- } \\
\text { dnia }\end{array}$ & $2 \mathrm{~h}$ & $3 \mathrm{~h}$ & $4 \mathrm{~h}$ & $\begin{array}{c}\text { mean } \\
\text { śre- } \\
\text { dnia }\end{array}$ & $2 \mathrm{~h}$ & $3 \mathrm{~h}$ & $4 \mathrm{~h}$ & $\begin{array}{c}\text { mean } \\
\text { śre- } \\
\text { dnia }\end{array}$ \\
\hline Myrcene - Mircen & $1.42 \pm 0.13$ & $1.37 \pm 0.15$ & $1.39 \pm 0.09$ & 1.40 & $0.62 \pm 0.12$ & $0.82 \pm 0.14$ & $0.78 \pm 0.19$ & 0.74 & 1.02 & 1.10 & 1.09 & 1.07 \\
\hline$\alpha$-Terpinene - $\alpha$-Terpinen & $2.04 \pm 0.08$ & $1.96 \pm 0.12$ & $1.93 \pm 0.09$ & 1.98 & $1.50 \pm 0.11$ & $1.69 \pm 0.12$ & $1.56 \pm 0.11$ & 1.59 & 1.77 & 1.83 & 1.75 & 1.78 \\
\hline p-Cymene - p-Cymen & $9.53 \pm 0.27$ & $8.76 \pm 0.35$ & $8.99 \pm 0.27$ & 9.10 & $5.06 \pm 0.46$ & $5.68 \pm 0.15$ & $5.41 \pm 0.22$ & 5.39 & 7.30 & 7.22 & 7.20 & 7.24 \\
\hline Eucalyptol - Eukaliptol & $1.65 \pm 0.08$ & $1.51 \pm 0.09$ & $1.32 \pm 0.05$ & 1.50 & $0.85 \pm 0.05$ & $0.83 \pm 0.04$ & $0.72 \pm 0.17$ & 0.80 & 1.25 & 1.17 & 1.02 & 1.15 \\
\hline $\mathrm{Y}$-Terpinene $-\mathrm{y}$-Terpinen & $8.67 \pm 0.35$ & $8.46 \pm 0.32$ & $8.42 \pm 0.29$ & 8.52 & $6.30 \pm 0.55$ & $6.84 \pm 0.57$ & $6.62 \pm 0.56$ & 6.59 & 7.49 & 7.65 & 7.52 & 7.55 \\
\hline $\begin{array}{l}\text { cis-Sabinene hydrate } \\
\text { cis Hydrat sabinenu }\end{array}$ & $1.17 \pm 0.08$ & $1.17 \pm 0.12$ & $1.03 \pm 0.03$ & 1.12 & $1.48 \pm 0.18$ & $1.38 \pm 0.10$ & $1.39 \pm 0.13$ & 1.42 & 1.33 & 1.28 & 1.21 & 1.27 \\
\hline $\begin{array}{l}\text { Thymoquinone } \\
\text { Tymochinon }\end{array}$ & $2.01 \pm 0.49$ & $1.95 \pm 0.04$ & $2.25 \pm 0.10$ & 2.07 & $0.44 \pm 0.08$ & $0.47 \pm 0.15$ & $0.65 \pm 0.26$ & 0.52 & 1.23 & 1.21 & 1.45 & 1.30 \\
\hline Carvacrol - Karwakrol & $57.10 \pm 2.57$ & $56.55 \pm 1.69$ & $55.20 \pm 0.60$ & 56.28 & $68.53 \pm 0.76$ & $64.28 \pm 1.94$ & $63.39 \pm 1.73$ & 65.40 & 62.82 & 60.42 & 59.30 & 60.84 \\
\hline $\begin{array}{l}\beta \text {-Caryophyllene } \\
\beta \text {-Kariofilen }\end{array}$ & $4.58 \pm 0.20$ & $5.09 \pm 0.24$ & $5.54 \pm 0.03$ & 5.07 & $4.94 \pm 0.21$ & $5.32 \pm 0.03$ & $5.59 \pm 0.13$ & 5.28 & 4.76 & 5.21 & 5.57 & 5.18 \\
\hline$\beta$-Bisabolene $-\beta$-Bisabolen & $0.75 \pm 0.26$ & $1.03 \pm 0.25$ & $1.29 \pm 0.04$ & 1.02 & $0.43 \pm 0.16$ & $0.72 \pm 0.20$ & $0.88 \pm 0.25$ & 0.68 & 0.59 & 0.88 & 1.09 & 0.85 \\
\hline$\delta$-Cadinene - $\delta$-Kadinen & $0.58 \pm 0.04$ & $0.71 \pm 0.05$ & $0.78 \pm 0.02$ & 0.69 & $0.76 \pm 0.04$ & $0.96 \pm 0.19$ & $1.10 \pm 0.09$ & 0.94 & 0.67 & 0.84 & 0.94 & 0.82 \\
\hline $\begin{array}{l}\text { Caryophyllene oxide } \\
\text { Tlenek kariofilenu }\end{array}$ & $1.20 \pm 0.16$ & $1.25 \pm 0.08$ & $1.32 \pm 0.07$ & 1.26 & $0.54 \pm 0.02$ & $0.56 \pm 0.04$ & $0.56 \pm 0.05$ & 0.55 & 0.87 & 0.91 & 0.94 & 0.91 \\
\hline $\begin{array}{l}\text { Linolenic acid } \\
\text { Kwas linolenowy }\end{array}$ & $0.30 \pm 0.26$ & $0.71 \pm 0.09$ & $0.84 \pm 0.06$ & 0.62 & - & $0.74 \pm 0.22$ & $1.31 \pm 0.20$ & 0.68 & 0.15 & 0.73 & 1.08 & 0.65 \\
\hline Mean - Średnia & 7.00 & 6.97 & 6.95 & 6.97 & 7.04 & 6.95 & 6.92 & 6.97 & 7.02 & 6.95 & 6.93 & 6.97 \\
\hline $\begin{array}{l}\text { LSD }_{\alpha=0.05} \text { for factor I } \\
\text { NIR }_{\alpha=0.05} \text { dla czynnika I }\end{array}$ & \multicolumn{4}{|c|}{1.482} & \multicolumn{4}{|c|}{1.574} & \multicolumn{4}{|c|}{1.019} \\
\hline $\begin{array}{l}\text { LSD }_{\alpha=0.05} \text { for factor II } \\
\text { NIR }_{\alpha=0.05} \text { dla czynnika II }\end{array}$ & \multicolumn{4}{|c|}{ n.s. } & \multicolumn{4}{|c|}{ n.s. } & \multicolumn{4}{|c|}{ n.s. } \\
\hline $\begin{array}{l}\text { LSD } \alpha=0.05 \text { for interaction I x I| } \\
\text { NIR }_{\alpha=0.05} \text { dla interakcji I x II }\end{array}$ & \multicolumn{4}{|c|}{ n.s. } & \multicolumn{4}{|c|}{0.740} & \multicolumn{4}{|c|}{0.644} \\
\hline
\end{tabular}

n.s. - not significant - różnice nieistotne.

\pm - standard deviation - średnia \pm odchylenie standardowe $(n=3)$. 
Table 5. Content of main essential oil constituents in winter savory herb in dependence on distillation time in Clevenger apparatus

Tabela 5. Zawartość głównych składników olejku eterycznego z cząbru górskiego w zależności od czasu destylacji w aparacie Clevengera

\begin{tabular}{|c|c|c|c|c|c|c|c|c|c|c|c|c|}
\hline \multirow{3}{*}{$\begin{array}{l}\text { Essential oil constituent } \\
\text { (factor I) } \\
\text { Składnik olejku } \\
\text { eterycznego } \\
\text { (czynnik I) }\end{array}$} & \multicolumn{12}{|c|}{ Distillation time (factor II) - Czas destylacji (czynnik II) } \\
\hline & \multicolumn{4}{|c|}{2014} & \multicolumn{4}{|c|}{2015} & \multicolumn{4}{|c|}{ 2014-2015 } \\
\hline & $2 \mathrm{~h}$ & $3 \mathrm{~h}$ & $4 \mathrm{~h}$ & $\begin{array}{l}\text { mean } \\
\text { śre- } \\
\text { dnia }\end{array}$ & $2 \mathrm{~h}$ & $3 \mathrm{~h}$ & $4 \mathrm{~h}$ & $\begin{array}{l}\text { mean } \\
\text { śre- } \\
\text { dnia }\end{array}$ & $2 \mathrm{~h}$ & $3 \mathrm{~h}$ & $4 \mathrm{~h}$ & $\begin{array}{c}\text { mean } \\
\text { śre- } \\
\text { dnia }\end{array}$ \\
\hline Myrcene - Mircen & $1.29 \pm 0.02$ & $1.30 \pm 0.12$ & $1.34 \pm 0.11$ & 1.31 & $0.59 \pm 0.14$ & $0.49 \pm 0.15$ & $0.47 \pm 0.03$ & 0.52 & 0.94 & 0.90 & 0.91 & 0.91 \\
\hline$\alpha$-Terpinene - $\alpha$-Terpinen & $1.88 \pm 0.08$ & $1.96 \pm 0.14$ & $1.90 \pm 0.09$ & 1.91 & $1.47 \pm 0.11$ & $1.27 \pm 0.13$ & $1.20 \pm 0.03$ & 1.31 & 1.68 & 1.62 & 1.55 & 1.61 \\
\hline p-Cymene-p-Cymen & $9.38 \pm 0.46$ & $9.23 \pm 0.17$ & $9.13 \pm 0.16$ & 9.25 & $5.05 \pm 0.60$ & $4.40 \pm 0.21$ & $4.79 \pm 0.12$ & 4.75 & 7.22 & 6.82 & 6.96 & 7.00 \\
\hline Eucalyptol - Eukaliptol & $1.43 \pm 0.25$ & $1.28 \pm 0.13$ & $1.53 \pm 0.13$ & 1.41 & $0.81 \pm 0.02$ & $0.80 \pm 0.05$ & $0.81 \pm 0.03$ & 0.81 & 1.12 & 1.04 & 1.17 & 1.11 \\
\hline $\mathrm{Y}$-Terpinene $-\mathrm{v}$-Terpinen & $8.22 \pm 0.15$ & $8.75 \pm 0.65$ & $8.30 \pm 0.38$ & 8.42 & $6.23 \pm 0.49$ & $5.49 \pm 0.68$ & $5.21 \pm 0.13$ & 5.65 & 7.23 & 7.12 & 6.76 & 7.03 \\
\hline $\begin{array}{l}\text { cis-Sabinene hydrate } \\
\text { cis Hydrat sabinenu }\end{array}$ & $1.15 \pm 0.09$ & $1.12 \pm 0.14$ & $1.06 \pm 0.11$ & 1.11 & $1.39 \pm 0.23$ & $1.49 \pm 0.17$ & $1.32 \pm 0.14$ & 1.40 & 1.27 & 1.31 & 1.19 & 1.26 \\
\hline $\begin{array}{l}\text { Thymoquinone } \\
\text { Tymochinon }\end{array}$ & $2.55 \pm 0.12$ & $2.06 \pm 0.46$ & $2.42 \pm 0.21$ & 2.34 & $0.74 \pm 0.13$ & $0.65 \pm 0.13$ & $0.80 \pm 0.01$ & 0.73 & 1.65 & 1.36 & 1.61 & 1.54 \\
\hline Carvacrol - Karwakrol & $56.65 \pm 0.64$ & $56.54 \pm 0.21$ & $54.44 \pm 1.04$ & 55.88 & $66.95 \pm 2.00$ & $66.06 \pm 1.66$ & $65.33 \pm 0.54$ & 66.11 & 61.80 & 61.30 & 59.89 & 61.00 \\
\hline $\begin{array}{l}\beta \text {-Caryophyllene } \\
\beta \text {-Kariofilen }\end{array}$ & $4.88 \pm 0.21$ & $5.09 \pm 0.12$ & $5.52 \pm 0.07$ & 5.16 & $5.26 \pm 0.07$ & $5.74 \pm 0.27$ & $5.77 \pm 0.05$ & 5.59 & 5.07 & 5.42 & 5.65 & 5.38 \\
\hline $\begin{array}{l}\beta \text {-Bisabolene } \\
\beta \text {-Bisabolen }\end{array}$ & $0.93 \pm 0.19$ & $1.00 \pm 0.13$ & $1.33 \pm 0.22$ & 1.09 & $0.60 \pm 0.14$ & $0.80 \pm 0.36$ & $0.98 \pm 0.13$ & 0.79 & 0.77 & 0.90 & 1.16 & 0.94 \\
\hline$\delta$-Cadinene - $\delta$-Kadinen & $0.65 \pm 0.04$ & $0.69 \pm 0.02$ & $0.81 \pm 0.04$ & 0.72 & $0.90 \pm 0.03$ & $1.01 \pm 0.13$ & $1.10 \pm 0.13$ & 1.01 & 0.78 & 0.85 & 0.96 & 0.86 \\
\hline $\begin{array}{l}\text { Caryophyllene oxide } \\
\text { Tlenek kariofilenu }\end{array}$ & $1.38 \pm 0.07$ & $1.16 \pm 0.15$ & $1.36 \pm 0.12$ & 1.30 & $0.52 \pm 0.04$ & $0.61 \pm 0.18$ & $0.65 \pm 0.08$ & 0.59 & 0.95 & 0.89 & 1.01 & 0.95 \\
\hline $\begin{array}{l}\text { Linolenic acid - } \\
\text { Kwas linolenowy }\end{array}$ & $0.59 \pm 0.17$ & $0.60 \pm 0.15$ & $0.80 \pm 0.12$ & 0.66 & $0.85 \pm 0.16$ & $1.36 \pm 0.60$ & $1.78 \pm 0.16$ & 1.33 & 0.72 & 0.98 & 1.29 & 1.00 \\
\hline Mean - Średnia & 7.00 & 6.99 & 6.92 & 6.97 & 7.03 & 6.94 & 6.94 & 6.97 & 7.01 & 6.96 & 6.93 & 6.97 \\
\hline $\begin{array}{l}\text { LSD }_{\alpha=0.05} \text { for factor I } \\
\text { NIR }_{\alpha=0.05} \text { dla czynnika I }\end{array}$ & \multicolumn{4}{|c|}{0.776} & \multicolumn{4}{|c|}{1.178} & \multicolumn{4}{|c|}{0.664} \\
\hline $\begin{array}{l}\text { LSD }_{\alpha=0.05} \text { for factor II } \\
\text { NIR }_{\alpha=0.05} \text { dla czynnika II }\end{array}$ & \multicolumn{4}{|c|}{ n.s. } & \multicolumn{4}{|c|}{ n.s. } & \multicolumn{4}{|c|}{ n.s. } \\
\hline $\begin{array}{l}\text { LSD }_{\alpha=0.05} \text { for interaction I x II } \\
\text { NIR } \alpha=0.05 \text { dla interakcji I x I| }\end{array}$ & \multicolumn{4}{|c|}{0.570} & \multicolumn{4}{|c|}{ n.s. } & \multicolumn{4}{|c|}{0.611} \\
\hline
\end{tabular}

Explanations see Table 4 - Objaśnienia zob. tab. 4. 
Analysis of the results obtained using the Deryng apparatus, in the year 2015 and for the synthesis of both years of the study, showed a significant interaction for carvacrol, $\beta$-caryophyllene and linolenic acid. For carvacrol the most effective was a 2 hour distillation time, less effective was 3 hours and the least effective was 4 hours. On the other hand, significantly higher amounts of $\beta$-caryophyllene and linolenic acid were obtained when samples were distilled for 3 and 4 hours than for 2 hours. Linolenic acid was not detected using a 2 hour distillation time in the year 2015. According to the Clevenger apparatus this interaction was significant in the first year of the study and the synthesis of both of the study years, and the results were similar to the ones obtained for the Deryng apparatus. Significantly higher amounts of carvacrol were noted for 2 and 3 hour distillation in the Clevenger apparatus, while for $\beta$-caryophyllene were 3 and 4 hours, in 2014 . The synthesis of the two year results confirmed that a 2 and 3 hour distillation time was more effective on the content of carvacrol than a 4 hour distillation.

\section{DISCUSSION}

The results obtained are similar to the data from the literature mentioning carvacrol, p-cymene and $\mathrm{y}$-terpinene as major components in Satureja montana L. essential oil (Piccaglia et al. 1991; Miladi et al. 2013; Haloci et al. 2014; Garcia-Rellan et al. 2015; Trifan et al. 2015).

The essential oil isolated from winter savory grown in northern Italy (Piccaglia et al. 1991) contained carvacrol (26.38-41.23\%), p-cymene (11.00-16.32\%) and y-terpinene (1.40-6.16\%) as main constituents. Plants collected in the south of France (Miladi et al. 2013) produced oil dominated by carvacrol (53.35\%), y-terpinene (13.54\%) and p-cymene (13.03\%). Also, essential oil derived from plants cultivated in northeastern Romania (Trifan et al. 2015) was dominated by carvacrol $(63.40 \%)$, p-cymene $(10.97 \%)$ and $y$-terpinene $(3.70 \%)$. Rich in carvacrol $(59.72 \%)$ and $y$-terpinene $(17.40 \%)$ was essential oil isolated from Spanish savory (Garcia-Rellan et al. 2015), while S. montana collected in north of Albania (Haloci et al. 2014) shows large variations in the content of carvacrol (6.11-67.68\%), p-cymene (5.90-20.86\%) and $y$-terpinene $(2.05-8.45 \%)$ in the volatile oil.

Michaelakis et al. (2007) reported as major components of Greek savory carvacrol (2.27-55.42\%), p-cymene (5.48-10.39\%), y-terpinene (6.49-20.12\%) and $\beta$-caryophyllene (3.68-5.47\%), while Dardioti (2005) stated that linalool dominates (> 23\%) in all essential oils obtained from plants collected on the territory of Greece.

In contrast, the S. montana volatile oil estimated by Cavar et al. (2008) was characterized by high content of geraniol (22.30\%) and terpinen-4-ol (10.30\%).

The amount of $\beta$-caryophyllene (4.58-5.77\%) found in our oils was comparable with the results obtained by Michaelakis et al. (2007). However, the content of linalool $(0.18-0.35 \%)$ and terpinene-4-ol $(0.58-0.82 \%)$ was much lower as compared with results published by Dardioti (2005) and Cavar et al. (2008).

Generally, such large differences in the chemical composition may be explained by the occurrence of various Satureja montana essential oil chemotypes (Milos et al. 2001). According to Cavar et al. (2008) the prevailing carvacrol/thymol chemotypes determine 
strong flavor of the plants. Moreover, they exhibit a significant antioxidant potential, while the linalool or geraniol chemotypes have a lower activity.

The comparison of different extraction techniques used for the isolation of $S$. montana essential oil has been reported by several authors (Grosso et al. 2007; Coelho et al. 2012; Rzepa et al. 2012; Damjanovic-Vratnica et al. 2016).

Supercritical fluid extraction (SFE) of the volatile oil of the aerial parts of winter savory carried out in different conditions of pressure, temperature and $\mathrm{CO}_{2}$ flow rate were compared with those obtained by hydrodistillation (HD) in Clevenger apparatus (Grosso et al. 2007). The main compounds detected in the oils were carvacrol (41-64\%), p-cymene (6-18\%), thymol (8-11\%), $\gamma$-terpinene $(2-9 \%)$ and $\beta$-bisabolene (2-4\%). The major difference between these two technique was relative percentage of thymoquinone $(0.17 \%$ for HD vs. $3.07 \%$ for SFE). Similarly, the savory oils obtained by SFE and HD were investigated by Coelho et al. (2012). Carvacrol (53\% in both cases), thymol (11\% in both cases), p-cymene (12.8\% for HD vs. $10.1 \%$ for SFE), $\mathrm{Y}$-terpinene $(8.9 \%$ for HD vs. $4.3 \%$ for SFE) and $\beta$-bisabolene ( $2 \%$ for HD vs. $2.5 \%$ for SFE) were the main constituents. Also, in these case the major difference was the relative amount of thymoquinone $(0.2 \%$ for HD vs. $2.9 \%$ for SFE). Results published by Damjanovic-Vratnica et al. (2016) indicated that the major compounds in winter savory oil obtained by $\mathrm{SC}-\mathrm{CO}_{2}$ extraction from differently treated matrix and HD were: thymol (30.4-35.4 and 35.3\%), carvacrol (11.5-14.1 and 14.1\%), y-terpinene (10.2-11.4 and $9.1 \%)$ and p-cymene (8.3-10.1 and 8.6\%), respectively. Interestingly, that the major differences between these two technique $\left(\mathrm{SC}-\mathrm{CO}_{2}\right.$ and $\left.\mathrm{HD}\right)$ was the occurrence of thymoquinone (0.3-1.4 and $0 \%)$.

In the present study, the percentage of thymoquinone was almost equal in the savory oils obtained by hydrodistillation using two different distillation apparatuses $(0.44-2.25 \%$ for Deryng and $0.65-2.55 \%$ for Clevenger, respectively).

Studies conducted by Rzepa et al. (2012) focused on a comparison of the efficiency of the methods used for derivation and fingerprinting of the volatile fraction from winter savory. The compared techniques were the headspace gas chromatography with mass spectrometric detection run at two different headspace temperatures and the distillation in Deryng and Clevenger apparatus. They revealed that the headspace extraction carried out at $100^{\circ} \mathrm{C}$ was more effective for isolation of volatile compounds than distillation. Moreover, in the essential oil isolated with use of Clevenger apparatus only four compounds were identified (1-octen-3-ol, p-cymene, y-terpinene, and carvacrol), while in the essential oil isolated with use of Deryng apparatus, six additional compounds ( $\alpha$-thujene, camphene, $\beta$-myrcene, $\alpha$-terpinene, $\beta$-terpineol and thymoquinone) were found.

Our results are opposite to these findings. The number of compounds identified in winter savory oils isolated by use of two different distillation apparatuses was the same. Only, in case of few constituents the time of distillation had a significant effect on their percentage content in the essential oil.

The lack of differences in the number of chemical compounds presented in the essential oils isolated from the flowers of Mutelina purpurea Thell. with use of Deryng and Clevenger apparatuses were found by Baj et al. (2015). In both essential oils (EOs) 58 identical compounds were detected. Sabinene, a-pinene, myrcene, (E)-sesquisabinene hydrate, 
(Z)-sesquisabinene hydrate and $\alpha$-bisabolol dominated in Deryng as well as Clevenger-EOs. Interestingly, there were also small quantitative differences between the main essential oils components observed. Only the content of myrcene was different in both oils.

The compared distillation apparatuses differ in their construction, what probably affects the yield and percentage composition of essential oils.

The influence of distillation time on the yield and composition of essential oils is still widely investigated. The number of reports concerning with this topic increases year by year.

According to the most literature data, the distillation of the essential oil is carried out for 2 to 4 hours. However, Garzoli et al. (2015) stated that the time of essential oil extraction depends on the purpose of the study.

Based on these findings it can be concluded that the choice of distillation time is related to the final destination of essential oil. Each essential oil, which may be used medicinally should meet requirements regarding the composition of major components. Moreover, the amounts of active constituents in the oil may change during distillation.

\section{CONCLUSIONS}

This study revealed that the essential oils obtained from winter savory herb using two different distillation apparatuses: Deryng and Clevenger had similar compositions.

In case of use Deryng apparatus, for high carvacrol content in the volatile oil, the most effective was 2 hour distillation and the least effective - 4 hour distillation. In case of Clevenger apparatus, significantly higher amounts of carvacrol were noted for 2 and 3 hour distillation than for 4 hours.

\section{REFERENCES}

Baj T., Sieniawska E., Kowalski R., Wesolowski M., Ulewicz-Magulska B. 2015. Effectivness of the Deryng and Clevenger-type apparatus in isolation of various types of components of essential oil from the Mutelina Purpurea Thell. flowers. Acta Pol. Pharm. Drug Res. 72(3), 505-515.

Cavar S., Maksimovic M., Solic M.E., Jerkovic-Mujkic A., Basta R. 2008. Chemical composition and antioxidant and antimicrobial activity of two Satureja essential oils. Food Chem. 111, 648-653.

Chorianopoulos N., Kalpoutzakis E., Aligiannis N., Mitaku S., Nychas G.J., Haroutounian S.A. 2004. Essential oils of Satureja, Origanum, and Thymus species: chemical composition and antibacterial activities against foodborne pathogens. J. Agric. Food Chem. 52(2), 8261-8267.

Ciani M., Menghini L., Mariani F., Pagiotti R., Menghini A., Fatichenti F. 2000. Antimicrobial properties of essential oil of Satureja montana L. on pathogenic and spoilage yeasts. Biotechnol. Lett. 22(12), 1007-1010.

Coelho J.P., Cristino A.F., Matos P.G., Rauter A.P., Nobre B.P., Mendes R.L., Barroso J.G., Mainar A., Urieta J.S., Fareleira J.M.N.A., Sovova H., Palavra A.F. 2012. Extraction of volatile oil from aromatic plants with supercritical carbon dioxide: experiments and modeling. Molecules 17 , 10550-10573.

Damjanovic-Vratnica B., Perovic A., Sukovic D., Perovic S. 2011. Effect of vegetation cycle on chemical content and antibacterial activity of Satureja montana L. Arch. Biol. Sci., Belgrade 63(4), 1173-1179.

Damjanovic-Vratnica B., Perovic S., Lu T., Santos R. 2016. Effect of matrix pretreatment on the supercritical $\mathrm{CO}_{2}$ extraction of Satureja montana essential oil. Chem. Ind. Chem. Eng. Q. 22(2), 201-209. 
Dardioti A. 2005. Biosystematic study of Satureja montana L. group in Greece. Doctorate Thesis, Thessaloniki, Aristotle University of Thessaloniki (AUTH), Greece. [typescript]

European Pharmacopoeia 5.0. 2004. Strasbourg, Council of Europe, 217-218.

Filipiak K., Wilkos S. 1995. Obliczenia statystyczne. Opis systemu AWAR. Puławy, IUNG, 324, 1-52. [in Polish]

Garcia-Rellan D., Blazquez M.A., Boira H. 2015. Differential essential oil composition and morphology between perennial Satureja species growing in Spain. Rec. Nat. Prod. 9(4), 623-627.

Garzoli S., Pirolli A., Vavala E., Di Sotto A., Sartorelli G., Bozovic M., Angiolella L., Mazzanti G., Pepi F., Ragno R. 2015. Multidisciplinary approach to determine the optimal time and period for extracting the essential oil from Mentha suaveolens Ehrh. Molecules 20, 9640-9655.

Grosso C., Cardoso M.A.T., Figueiredo A.C., Moldao-Martins M., Burillo J., Urieta J.S., Barroso J.G., Coelho J.A., Palavra A.M. 2007. Supercritical fluid extraction, hydrodistillation and Soxhlet extraction of the aerial part of winter savory. Comparative evaluation of the extraction method on the chemical composition, in: Proceedings of European Congress of Chemical Engineering (ECCE-6), Copenhagen, September 16-20, 2007, [b.w.], 1-5.

Haloci E., Toska V., Baldisserotto A., Goci E., Vertuani S., Manfredini S. 2014. Evaluation of antifungal activity of Satureja montana essential oil before and after inclusion in beta-cyclodextrine. Int. J. Pharm. Pharm. Sci. 6(7), 189-191.

Hassanein H.D., Said-Al Ahl H.A.H., Abdelmohsen M.M. 2014. Antioxidant polyphenolic constituents of Satureja montana L. growing in Egypt. Int. J. Pharm. Pharm. Sci. 6(4), 578-581.

Lawless J. 2002. The encyclopedia of essential oils. London, Thorsons Publishing Group, 170.

Lawrence B.M. 1979. Essential oils. Wheaton, IL, Allured Publishing Corporation.

Madsen H.L., Nielsen B.R.R., Bertelsen G., Skibsted L.H. 1996. Screening of antioxidative activity of spices. A comparison between assay based on ESR Trapping and Electrochemical measurement of oxygen consumption. Food Chem. 57, 331-337.

Michaelakis A., Theotokatos S.A., Koliopoulos G., Chorianopoulos N.G. 2007. Essential oils of Satureja species: insecticidal effect on Culex pipiens Larvae (Diptera: Culicidae). Molecules 12, 2567-2578.

Miladi H., Slama R.B., Mili D., Zouari S., Bakhrouf A., Ammar E. 2013. Chemical composition and cytotoxic and antioxidant activities of Satureja montana L. essential oil and its antibacterial potential against Salmonella spp. strains. J. Chem. 2013, https://www.hindawi.com/journals/jchem/2013/275698/. DOI:10.1155/2013/275698.

Milos M., Radonic A., Bezic N., Dunkic V. 2001. Localities and seasonal variations in the chemical composition of essential oils of Satureja montana L. and Satureja cuneifolia ten. Flavour Fragr. J. 16, 157-160.

Pepeljak S., Stanic G., Potocki P. 1999. Antimicrobial activity of the ethanolic extract of Satureja montana ssp. montana. Acta Pharm. 49, 65-69.

Piccaglia R., Marotti M., Galletti G. 1991. Characterization of essential oil from a Satureja montana L. chemotype grown in Northern Italy. J. Essent. Oil Res. 3(3), 147-152.

Polish Pharmacopoeia VI. 2002. Warszawa, Urząd Rejestracji Produktów Leczniczych, Wyrobów Medycznych i Produktów Biobójczych ,151. [in Polish]

Polish Pharmacopoeia VIII. 2008. Warszawa, Urząd Rejestracji Produktów Leczniczych, Wyrobów Medycznych i Produktów Biobójczych, 229-230. [in Polish]

Radonic A., Milos M. 2003. Chemical composition and in vitro evaluation of antioxidant effect of free volatile compounds from Satureja montana L. Free Radic. Res. 37(6), 673-679.

Ruberto G., Baratta M.T. 2000. Antioxidant activity of selected essential oil components in two lipid model systems. Food Chem. 69, 167-174.

Rzepa J., Sajewicz M., Baj T., Gorczyca P., Włodek M., Głowniak K., Wksmundzka-Hajnos M., Kowalska T. 2012. A comparison of methodical approaches to fingerprinting of the volatile fraction from winter savory (Satureja montana). Chromatogr. Res. Int. 2012, https://www.hindawi.com/ /archive/2012/596807/. DOI:10.1155/2012/596807. 
Serrano C., Matos O., Teixeira B., Ramos, C., Neng N., Nogueira J., Nunes M.L., Marques A. 2011. Antioxidant and antimicrobial activity of Satureja montana L. extracts. J. Sci. Food Agric. 91(9), 1554-1560.

Skocibusic M., Bezic N. 2004. Phytochemical analysis and in vitro antimicrobial activity of two Satureja species essential oils. Phytother. Res. 18, 967-970.

Small E., Deutsch G. 2001. Culinary herbs for short-season gardeners. Toronto, Ismant Peony Press, Canada, 158.

Trifan A., Aprotosoaie A.C., Brebu M., Cioanca O., Gille E., Hancianu M., Miron A. 2015. Chemical composition and antioxidant activity of essential oil from Romanian Satureja montana L. Farmacia 63(3), 413-416.

Valnet J. 1990. The practice of aromatherapy. Vermont, Healing Arts Press, 185-186.

Wesołowska A., Grzeszczuk M., Jadczak D. 2014. Influence of harvest term on the content of carvacrol, p-cymene, $\gamma$-terpinene and $\beta$-caryophyllene in the essential oil of Satureja montana. Not Bot. Hort. Agrobot. 42(2), 392-397.

Zavatti M., Zanoli P., Benelli A., Rivasi M., Baraldi C., Baraldi M. 2011. Experimental study on Satureja montana as a treatment for premature ejaculation. J. Ethnopharmacol. 133(2), 629-633.

Abstract. The aim of the studies conducted in 2014-2015 was to determine the influence of distillation apparatus as well as distillation time on the yield and chemical composition of winter savory (Satureja montana L.) essential oil. Isolation of the essential oil from S. montana herb, collected during flowering period, was carried out using two different distillation apparatuses: Deryng and Clevenger. Moreover, different distillation times (2, 3 and 4 hours) were also applied. The obtained essential oils were analyzed by gas chromatography-mass spectrometry (GC-MS). GC-MS analysis resulted in the identification of 47 compounds in the essential oils obtained in Deryng and Clevenger apparatus, respectively. Carvacrol (54.44-68.53\%) was the major constituent of all S. montana oil samples. Other important compounds were: $\mathrm{Y}$-terpinene (5.21-8.67\%), p-cymene (4.40-9.53\%), a-terpinene (1.20-2.04\%) and $\beta$-caryophyllene (4.58-5.77\%). On the basis of the obtained data it was proved that the time of distillation of $S$. montana herb in Deryng apparatus had no significant effect on its content. However, the amount of volatile oil obtained in Clevenger apparatus was significantly influenced by distillation time. Moreover, there was no significant effect of the distillation time on the amount of the main essential oil constituents obtained with both types of apparatuses. However, there was a significant interaction found between the constituent and the distillation time. 